\title{
Cephalometric Evaluation of Vertical Dimension of Occlusion in Varying Malocclusions
}

\author{
Isha Aggarwal ${ }^{1} \quad$ Anindita Mallik ${ }^{1,0}$ Sanjay Mittal ${ }^{1} \quad$ Mandeep Bhullar $^{1} \quad$ Divya Singla $^{1} \quad$ Merry Goyal $^{1}$ \\ ${ }^{1}$ Department of Orthodontics and Dentofacial Orthopedics, Bhojia \\ Address for correspondence Anindita Mallik, Room No. 409, \\ Dental College, Baddi, Himachal Pradesh, India \\ Girls Hostel, Bhojia Dental College and Hospital, Baddi 173205 , \\ Himachal Pradesh, India (e-mail: mallik.ani3@gmail.com).
}

\begin{abstract}
Keywords

- Moroccan

- Solan

- vertical dimension of occlusion
\end{abstract}

Introduction The aim of modern cephalometrics is to evaluate the relationship of skeletal and dental functional units of the face and to implement treatment to establish the position of the units horizontally and vertically. Establishing a correct occlusal vertical dimension is considered one of the most important aspects of facial esthetics for patients in need of orthodontic treatment.

Aim The aim of this study is to evaluate the vertical dimension of occlusion in varying dental malocclusions in Solan population.

Materials and Methods The sample consisted of pretreatment lateral cephalograms of 100 patients ( 50 Class I and 50 Class II div 1), aged 15 to 30 years; six angular and one linear parameters were measured to determine the vertical dimension of occlusion.

Results All the parameters (Frankfort-mandibular plane angle [FMA], Occl/Frankfort horizontal plane [FHA], angle of Y-axis, Occl/SN, GoGn/SN, and ANS-Me) were found to be decreased in Class I than in Class II div 1 malocclusion except (ANS-Xi-Pm). All the parameters were found to be statistically significant $(p<0.05)$ when compared between groups. When cephalometric norms of the present study were compared with Moroccan population, all parameters (ANS-Xi-Ptm, FMA, Occl/FH, Occl/SN, GoGn/SN, and ANS-Me) were found to be decreased in Solan population except angle of $\mathrm{Y}$-axis. All the parameters were clinically significant $(p<0.05)$ except ANS-Xi-Pm and FMA that were found to be clinically nonsignificant $(p>0.05)$.

Conclusion Solan population has distinct cephalometric characteristics, which should be used as the reference in future orthodontic treatments.

\section{Introduction}

The aim of modern cephalometrics is to evaluate the relationship of skeletal and dental functional units of the face and to implement treatment to establish the position of the units horizontally and vertically (profit and field 2000) establishing a correct occlusal vertical dimension. Vertical dimension of occlusion (VDO) is defined as the height of the lower face when the dental arches are in centric occlusion and it is measured in between two arbitrary sagittal points located above the upper lip and beneath the lower lip according to Batarec. ${ }^{1}$ Specifically, it is the height of the lower face when

\section{received}

July 24, 2019

accepted

July 30, 2019

published online

October 18, 2019 the dental arches are in maximum intercuspation. ${ }^{2}$ The loss of VDO leads to unavoidable pathogenesis in respect to the esthetics, functional efficacy, and structural balance. Several clinical situations such as tooth loss, posterior occlusal collapse, periodontal diseases, and occasionally bruxism require VDO correction.

Correct determination of VDO is of utmost importance for the functional and physiognomic rehabilitation of patients. Current knowledge on the morphological and functional aspects of maxillofacial complex, knowledge on genetic complexity of certain morphological structures, knowledge on inseparable association between morphology and function,
License terms

Hospital affiliated to Himachal

Pradesh University 
and knowledge on individual course of neuromuscular processes within the system have necessitated a need to reconstruct the previous natural VDO as precisely as possible..$^{2-7}$ Various methods of determining VDO other than cephalometry are unfortunately not very reliable; thus, cephalometric techniques have the advantage of providing an initial numerical VDO assessment that can be clinically verified. ${ }^{8}$ They determine the correlation between certain craniofacial components (points, lines, and/or angles), which remain relatively unchanged after tooth loss., ${ }^{2,9}$ Several studies have been performed within various ethnicities ${ }^{10-12}$ with the objective of establishing adequate cephalometric standards for reference, to guide the orthodontic therapeutics and prosthetic rehabilitation.

Vertical dimension is the length of the face as determined by the amount of separation of the jaws. There are two vertical dimensions. One is the length of the face when the teeth are in contact and the mandible is in centric relation (occlusal vertical dimension) and the other is the length of the face when the teeth are separated and the mandible is in a physiologic rest position (vertical dimension of rest). Both vertical dimensions are subject to change resulting from loss of teeth. ${ }^{9}$

\section{Aims and Objectives}

The aim of this study is to evaluate the VDO in varying dental malocclusions and to establish cephalometric norms for Solan population.

\section{Objectives}

The objectives of this article are as follows:

1. To evaluate VDO in Angel Class I occlusion.

2. To evaluate VDO in Angel Class II div 1 malocclusion.

3. To compare the cephalometric norms of present study with other population.

\section{Materials and Methods}

A total of 100 pretreatment lateral cephalograms of the patients who reported for fixed orthodontic treatment were taken from the archives of Department of Orthodontics and Dentofacial Orthopaedics, Bhojia Dental College, Himachal Pradesh. The patients were selected on the basis of following criteria.

\section{Inclusion Criteria}

1. Patients with complete set of permanent dentition irrespective of third molars.

2. Patients with Class I occlusion with mild or no crowding nor overbite exceeding $6 \mathrm{~mm}$, little or no Bjork's mandibular rotation.

3. Patients with Class II div 1 malocclusion.

\section{Exclusion Criteria}

1. Missing permanent teeth.

2. Patients with craniofacial anomalies.

3. Patients with previous history of orthodontic treatment.

The sample was divided into two groups with 50 subjects each on the basis of Angel system of classification (- Table 1).

All lateral cephalograms obtained were traced manually by the same investigator. The cephalometric landmarks were identified and marked (-Table 2; - Fig. 1). The analysis as proposed by Steiner, Tweed, Brodie, Wylie, and Ricketts was used to determine the VDO.

Various angular and linear measurements were evaluated to determine the VDO (- Table $\mathbf{3}$; - Figs. 2 and $\mathbf{3}$ ).

Table 1 Grouping of sample

\begin{tabular}{|l|l|}
\hline Group A & Group B \\
\hline $\begin{array}{l}\text { Angel Class I occlusion } \\
(n=50)\end{array}$ & $\begin{array}{l}\text { Angel Class II div 1 malocclusion } \\
(n=50)\end{array}$ \\
\hline
\end{tabular}

Table 2 Landmarks used in the study

\begin{tabular}{|l|l|l|}
\hline S. No. & Landmarks & Definition \\
\hline 1. & Sella & Defined as the constructed midpoint of the hypophyseal fossa \\
\hline 2. & Nasion & The most anterior point of the nasofrontal suture in the median plane \\
\hline 3. & Pm & $\begin{array}{l}\text { Point selected at the anterior border of the symphysis between point B and pogonion } \\
\text { where the curvature changes from concave to convex }\end{array}$ \\
\hline 4. & Anterior nasal spine (ANS) & Tip of the bony anterior nasal spine in the median plane \\
\hline 6. & Menton (Me) & $\begin{array}{l}\text { Menton is the most caudal point in the outline of the symphysis, regarded as the lowest } \\
\text { point of the mandible }\end{array}$ \\
\hline 7. & Gonion (Go) & $\begin{array}{l}\text { Brodie is located by taking the midpoint between the most anterior and inferior points } \\
\text { on the body of the chin }\end{array}$ \\
\hline 8. & Xi point & $\begin{array}{l}\text { Constructed point, intersection of the lines tangent to the posterior margin of the } \\
\text { ascending ramus and the mandibular base } \\
\text { Brodie is obtained by bisecting the angle formed by tangents to the lower and posterior } \\
\text { borders of the mandible }\end{array}$ \\
\hline
\end{tabular}

Note: See - Fig. 1 for more details. 


\section{Statistical Analysis}

The values so obtained were subjected to statistical analysis using SPSS 20 software. Mean and standard deviations were calculated for all the parameters for both the groups. To analyze the mean of parameters, comparison between the groups was done using paired t-test.

\section{Results}

The present study was conducted to evaluate VDO in varying dental malocclusions and to establish cephalometric norms in Solan population. The study included pretreatment lateral cephalograms of 100 patients divided into two Groups, that is, Group A (Class I, $n=50$ ) and Group B (Class II div 1 , $n=50$ ) on the basis of Angel system of classification. The VDO for both the groups was evaluated using six angular

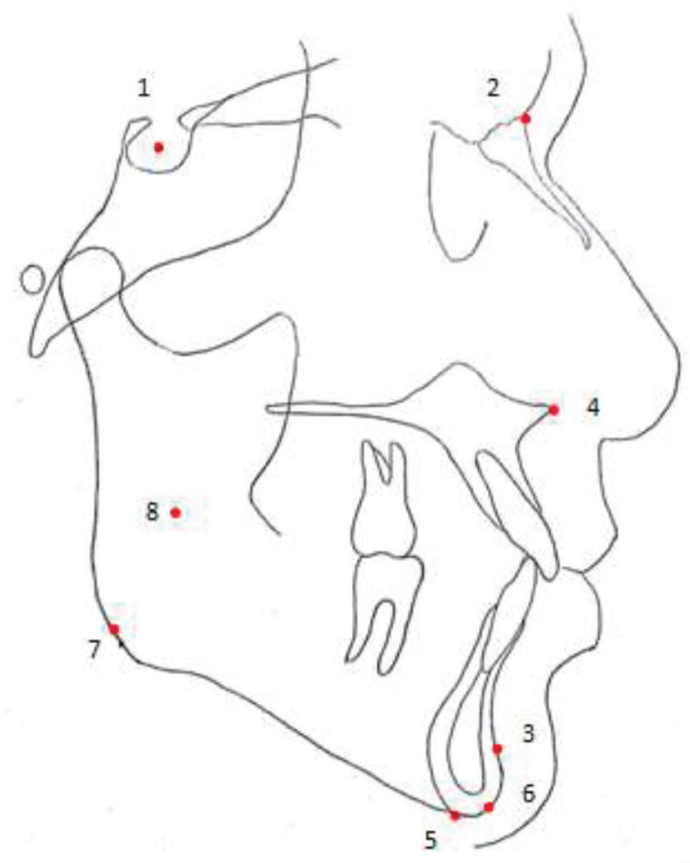

Fig. 1 Landmarks used in the study (see $\mathbf{- T a b l e} 2$ for more details). and one linear measurements. The sample in the present study was normally distributed as depicted in -Fig. 4. The mean and standard deviations of angular and linear measurements for Group A and Group B are depicted in - Tables 4 and 5.

\section{Angular Measurements}

In Group A (Class I), the mean of ANS-Xi-Pm was (45.94 \pm 2.17 ), whereas in Group B it was found to be smaller (44.480 \pm 2.85$)$. The means of FMA, Occl/FH, angle of Y-axis, Occl/SN, and GoGn/SN were found to be smaller in Group $A$, that is, $(25.6 \pm 1.90),(6.12 \pm 2.50),(62.22 \pm 4.14),(15.22 \pm 2.56)$, $(29.220 \pm 2.27)$, and in Group B the values were found to be greater $(29.720 \pm 2.40),(15.040 \pm 1.91),(64.400 \pm 2.48)$, (23.540 \pm 2.2$)$, (32.26 \pm 3.69$)$, respectively as depicted in - Tables 4 and 5.

\section{Linear Measurement}

In Group A, the mean of ANS-Me was found to be $54.6 \pm$ $3.36 \mathrm{~mm}$, whereas in Group $B$, it was found to be greater $(61.88 \pm 2.48 \mathrm{~mm})$ as depicted in - Tables 4 and $\mathbf{5}$.

\section{Comparison of Angular and Linear Measurements between the Groups}

When means of the cephalometric parameters were analyzed by comparing between the two groups as depicted in - Table 6, it showed that all the six angular measurements and one linear measurement were found to be clinically significant $(p>0.05)$.

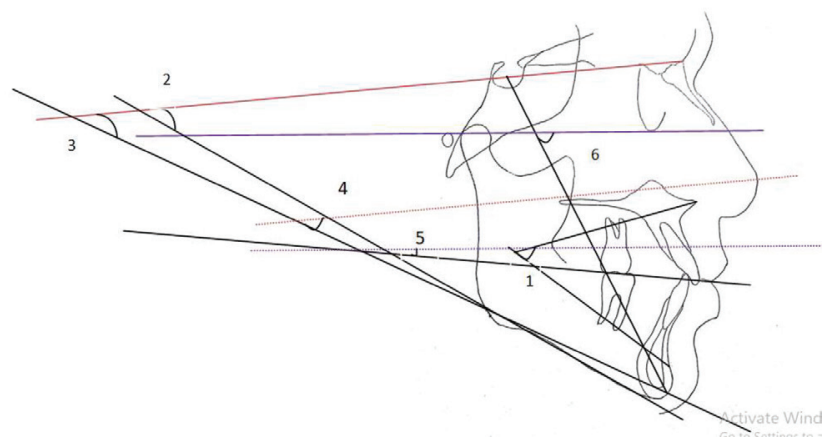

Fig. 2 Angular measurements used in the study.

Table 3 Angular and linear measurements used in the study

\begin{tabular}{|l|l|l|}
\hline S. No. & Measurements & Definitions \\
\hline 1. & ANS-Xi-Pm & This angle is formed by joining plane ANS-Xi and Xi-Pm $\left(46.2^{\circ} \pm 4.4^{\circ}\right)$ \\
\hline 2. & FMA & This angle is formed by joining mandibular plane with Frankfort horizontal plane $\left(25.6^{\circ} \pm 5.1^{\circ}\right)$ \\
\hline 3. & GoGn/SN & This angle is obtained by joining SN plane and mandibular plane $(G o-G n),\left(35.4 \pm 5.3^{\circ}\right)$ \\
\hline 4. & Occ/SN & This angle is obtained by joining the occlusal plane with SN plane $\left(19.3^{\circ} \pm 4.7^{\circ}\right)$ \\
\hline 5. & Occ/FH & Angle is obtained by joining occlusal plane with Frankfort horizontal plane $\left(9.1^{\circ} \pm 4^{\circ}\right)$ \\
\hline 6. & Angle of Y-axis & This angle is obtained by joining S-Me plane and Frankfort horizontal plane $\left(59.2^{\circ} \pm 3.4^{\circ}\right)$ \\
\hline 7. & ANn/FH & It is the linear distance measured between ANS to Me $(69.7 \pm 5.9 \mathrm{~mm})$ \\
\hline
\end{tabular}

Note: See $\mathbf{- F i g s .} \mathbf{2}$ and $\mathbf{3}$ for more details. 


\section{Comparison of Cephalometric Norms of Present Study with Established Cephalometric Standards}

When the means of the cephalometric norms (Class I) of the present study were compared with cephalometric norms (ClassI)ofMoroccan population, it was found that ANS-Xi-Pm (45.94 \pm 2.17$)$, FMA $(25.6 \pm 1.90)$, Occl/FH(6.12 \pm 2.50$)$, Occl/SN (15.22 \pm 2.56$), G o G n / S N(29.220 \pm 2.27)$, and

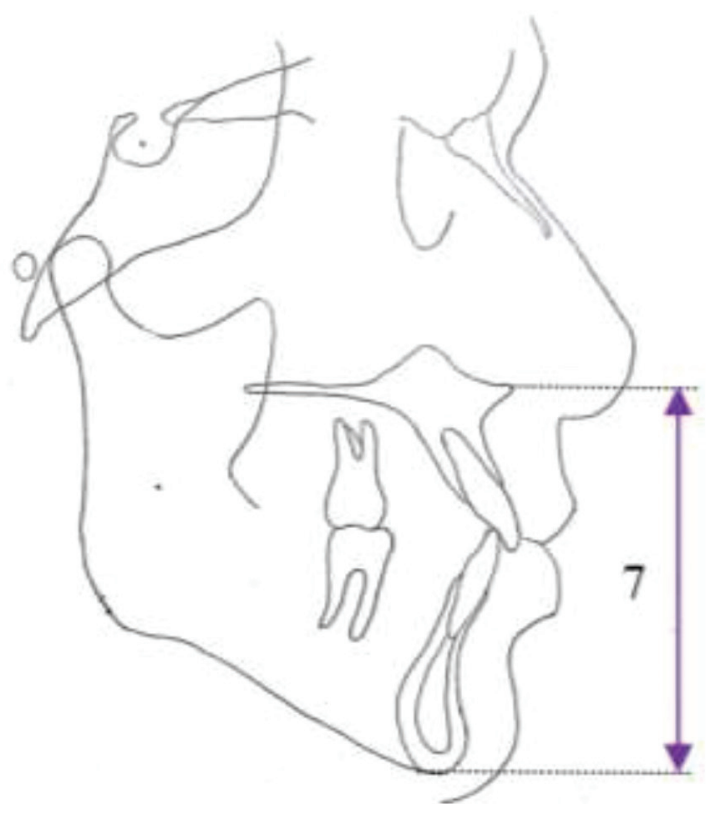

Fig. 3 Linear measurement used in the study (see - Table 3, 7th parameter).

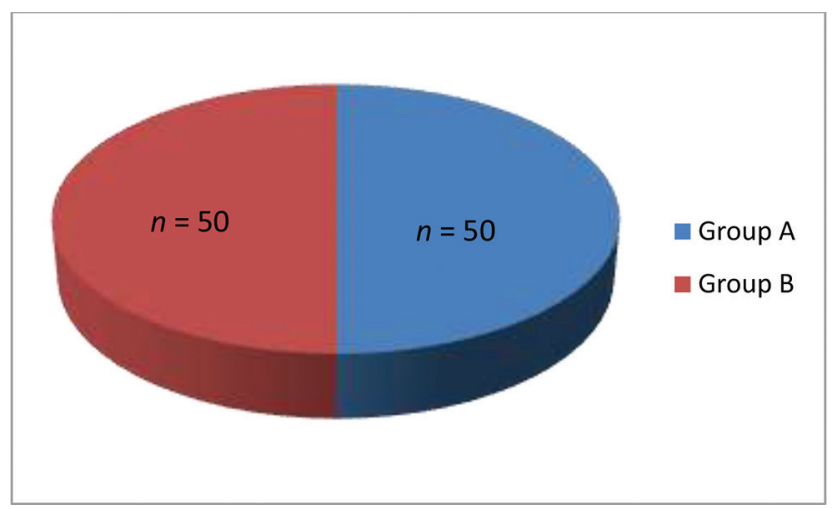

Fig. 4 Diagram showing equal distribution of sample in Group A (Class I occlusion, $n=50$ ) and Group B (Class II div 1 malocclusion, $n=50$ ).
ANS-Me (54.68 \pm 3.37$)$ were found to be smaller in Solan population, whereas in Moroccan population ANS-Xi-Pm $(46.2 \pm 4.4)$, FMA $(25.6 \pm 5.1)$, Occl/FH $(9.1 \pm 4)$, Occl/SN $(19.3 \pm 4.7)$, and $G o G n / S N(35.4 \pm 5.3)$ were found to be greater, respectively. The angle of Y-axis was increased in the Solan population $(62.22 \pm 4.14)$ than that of in the Moroccan population $(59.2 \pm 3.4)$. All the cephalometric parameters were found to be clinically significant $(p<0.05)$ except ANS-Xi-Pm and FMA $(p>0.05)$.

\section{Discussion}

In human beings, the lower face serves not only in the interest of digestion, speech, and respiration, but it also influences to a large extent the social acceptance and psychological well-being of the individual. ${ }^{14}$ Changes of the hard and soft tissues of the face and jaw complex following tooth loss and loss of support provided by natural occlusion lead to drastic changes in the vertical dimension of the lower face. Correct determination of VDO is of utmost importance for the functional and physiognomic rehabilitation of such patients. VDO assessment is a delicate stage within any orthodontic treatment. Therefore, it is necessary to identify objective parameters to achieve directions for optimal reconstruction of the VDO. Many clinical methods have been described for the determination of VDO, but no single method has proved superior. The use of cephalometric analysis represents one of the ways to identify better solutions in planning artificial occlusion complex. It specifically allows determination of the VDO in regard to those parts of craniofacial skeleton that remained unchanged after tooth loss. The aim of the present study was to establish precise cephalometric parameters of VDO, which could be used as objective guidelines in orthodontic therapy. The study included 100 pretreatment lateral cephalograms of the patients, aged 15 to 30 years who reported to the department for fixed mechanotherapy. The sample was then divided into two groups: Group A (Class I, $n=50$ ) and Group B (Class II div 1, $n=50$ ) on the basis of Angel system of classification. Six angular and one linear parameters were measured to determine the VDO.

The descriptive statistics showed that in Group A (Class I) all the parameters (FMA, Occl/FH, angle of Y-axis, Occl/SN, $\mathrm{GoGn} / \mathrm{SN}$, and ANS-Me) were found to be smaller than in Group B (Class II div 1) except for ANS-Xi-Pm. When the

Table 4 Descriptive statistics of vertical dimension of occlusion in Group A (Class I occlusion)

\begin{tabular}{|l|l|l|l|l|l|}
\hline Parameters & $\boldsymbol{n}$ & Mean & SD & Upper limit & Lower limit \\
\hline ANS-Xi-Pm & 50 & 45.94 & 2.17 & 49 & 41 \\
\hline FMA & 50 & 25.60 & 1.90 & 29 & 21 \\
\hline GoGn/SN & 50 & 29.22 & 2.28 & 33 & 26 \\
\hline Occ/SN & 50 & 15.22 & 2.56 & 19 & 13 \\
\hline Occ/FH & 50 & 6.12 & 2.50 & 9 & 4 \\
\hline Angle of Y-axis SGn/FH & 50 & 62.22 & 4.14 & 72 & 54 \\
\hline ANS-Me & 50 & 54.68 & 3.37 & 59 & 48 \\
\hline
\end{tabular}

Abbreviation: SD, standard deviation. 
Table 5 Descriptive statistics of vertical dimension of occlusion in Group B (Class II div 1 malocclusion)

\begin{tabular}{|l|l|l|l|l|l|}
\hline Parameters & $\boldsymbol{n}$ & Mean & SD & Upper limit & Lower limit \\
\hline ANS-Xi-Pm & 50 & 44.48 & 2.85 & 49 & 41 \\
\hline FMA & 50 & 29.72 & 2.41 & 33 & 27 \\
\hline GoGn/SN & 50 & 32.26 & 3.69 & 39 & 25 \\
\hline Occ/SN & 50 & 23.54 & 2.21 & 27 & 22 \\
\hline Occ/FH & 50 & 15.04 & 1.92 & 19 & 11 \\
\hline Angle of Y-axis SGn/FH & 50 & 64.40 & 2.48 & 69 & 61 \\
\hline ANS-Me & 50 & 61.88 & 2.48 & 60 & 49 \\
\hline
\end{tabular}

Abbreviation: SD, standard deviation.

Table 6 Comparison of parameters between Group A (Class I) and Group B (Class II div 1)

\begin{tabular}{|c|c|c|c|c|c|}
\hline Parameters & Malocclusion & Mean & SD & SE mean & $p$-Value \\
\hline \multirow[t]{2}{*}{ ANS-Xi-Pm } & Group A & 45.940 & 2.170 & 0.307 & 0.005 \\
\hline & Group B & 44.480 & 2.852 & 0.403 & \\
\hline \multirow[t]{2}{*}{ FMA } & Group A & 25.600 & 1.895 & 0.268 & 0.0001 \\
\hline & Group B & 29.720 & 2.408 & 0.341 & \\
\hline \multirow[t]{2}{*}{ Go Gn /SN } & Group A & 29.220 & 2.279 & 0.322 & 0.0001 \\
\hline & Group B & 32.260 & 3.691 & 0.522 & \\
\hline \multirow[t]{2}{*}{ Occ/SN } & Group A & 15.220 & 2.558 & 0.362 & 0.0001 \\
\hline & Group B & 23.540 & 2.206 & 0.312 & \\
\hline \multirow[t]{2}{*}{$\mathrm{Occ} / \mathrm{FH}$} & Group A & 6.120 & 2.496 & 0.353 & 0.0001 \\
\hline & Group B & 15.040 & 1.916 & 0.271 & \\
\hline \multirow[t]{2}{*}{ Angle of Y-axis SGn/FH } & Group A & 62.220 & 4.142 & 0.586 & 0.002 \\
\hline & Group B & 64.400 & 2.483 & 0.351 & \\
\hline \multirow[t]{2}{*}{ ANS-Me } & Group A & 54.680 & 3.365 & 0.476 & 0.0001 \\
\hline & Group B & 61.880 & 2.480 & 0.351 & \\
\hline
\end{tabular}

Abbreviations: SD, standard deviation; SE, standard error.

Table 7 Comparison of cephalometric norms of present study with Moroccan ${ }^{3}$ population

\begin{tabular}{|l|l|l|l|}
\hline Parameter & Solan population (Class I) & Moroccan population ${ }^{3}$ (Class I) & $p$-Value \\
\hline ANS-Xi-Pm & $45.94 \pm 2.17$ & $46.2 \pm 4.4$ & 0.664 \\
\hline FMA & $25.6 \pm 1.90$ & $25.6 \pm 5.1$ & 1 \\
\hline GoGn/SN & $29.22 \pm 2.28$ & $35.4 \pm 5.3$ & 0.0001 \\
\hline Occ/SN & $15.22 \pm 2.56$ & $19.3 \pm 4.7$ & 0.0001 \\
\hline Occ/FH & $6.12 \pm 2.50$ & $9.1 \pm 4$ & 0.0001 \\
\hline Angle of Y-axis SGn/FH & $62.22 \pm 4.14$ & $59.2 \pm 3.4$ & 0.0001 \\
\hline ANS-Me & $54.68 \pm 3.37$ & $69.7 \pm 5.9$ & 0.0001 \\
\hline
\end{tabular}

Note: Significant $(p<0.05)$.

Nonsignificant $(p>0.05)$.

means of all the parameters were compared between the two groups, they were found to be clinically significant $(p<0.05)$.

It is noteworthy that the increase in FMA and Y-axis angle describes a skeletal configuration that is typical of dolichocephaly, that is, with more protrusive position of nasomaxillary complex in relation to the mandible. ${ }^{15}$
All the values were larger in Group B than ANS-Xi-Pm, showing divergence of mandible and maxilla and vertical growth trend, and they are more prone to skeletal open bite. ${ }^{16,17}$

The VDO is the result of a musculoskeletal balance during growth. The skeletal morphology of the mandible is clearly related to the elevator muscles' organization. 
VDO is one of the determinants of prosthetic reconstruction, and cephalometric analysis can also help in decisions concerning the orientation of the occlusal plane, the curve of Spee, the anterior teeth positions, and the anterior guidance. $^{18}$

When the cephalometric norms of the present study were compared with the Moroccan population, it was found that the means of ANS-Xi-Pm and FMA were smaller in Solan population as compared with the Moroccan population and were found to be clinically insignificant $(p>0.05)$. The decrease in FMA angle in our population shows less pronounced facial heights than the Moroccan population. This is in accordance with a study by Lahlou et al ${ }^{16}$ The means of $\mathrm{Occl} / \mathrm{FH}, \mathrm{Occl} / \mathrm{SN}$, and $\mathrm{GoGn} / \mathrm{SN}$ were also found to be smaller in Solan population when compared with the Moroccans but were clinically significant $(p<0.05)$. Occ/SN is smaller in the present study suggestive of long face in Moroccan population as they have maxilla rotating downward. ${ }^{2,3,18} \mathrm{Occ} / \mathrm{FH}$ is larger in Moroccan population that can be due to Frankfurt plane inclination. GoGn/SN angle is smaller in Solan population when compared with Moroccan population showing vertical growth pattern and open bite in the Moroccans. ${ }^{3}$ However, the angle of Y-axis was increased in the Solan population when compared with the Moroccan population and it was found to be clinically significant $(p<0.05)$, stating more vertical growth pattern in Solan population; this is in concordance by Kharbanda et al. ${ }^{17}$ ANS-Me was found to be smaller in the Solan population when compared with the Moroccan population, reflecting that decreased lower facial heights can be attributed to the decrease in the size of mandible (Yemeni, Iraqi, Moroccan).

The literature review shows that there is no "magic" method for the evaluation of the VDO. The concept of a vertical comfort range is generally accepted and, in fact, for a patient, the real question is in which direction does the variation of VDO (increase or decrease) lead to the best harmony of its musculoskeletal determinants. It is easier to assess skeletal morphology than muscles. Therefore, cephalometric analysis, despite its imperfections, may help the practitioner to understand the direction of the treatment concerning the lower facial height in occlusion.

\section{Conclusion}

This study enabled us to establish cephalometric standards specific to Solan population. We also observed that Solan population exhibits higher amounts of VDO compared with international norms with decreased lower facial height.

Although our study has limitations, we suggest that dentists from this region adopt the system of cephalometric norms resulting from our investigation, as these values reflect the craniofacial morphology and esthetic profile of Solan population better than other (international) cephalometric norms. While this system would be applicable to Solan population, as a whole facial harmony existed both in men and women.

\section{Note}

This study was conducted in Bhojia Dental College and Hospital, Baddi, Himachal Pradesh, India

\section{Conflict of Interest}

None declared.

\section{References}

1 Batarec E. Lexicon of the terms of dental prosthesis. Julien Prelate, Paris; 1972

2 Orthlieb JD, Laurent M, Laplanche O. Cephalometric estimation of vertical dimension of occlusion. J Oral Rehabil 2000;27(9):802-807

3 Ousehal L, Jouhadi E, Bennani A. Vertical dimension of occlusion (VDO): cephalometric norms for a Moroccan population. J Orofac Orthop 2016;77(1):39-44

4 Ajayi EO. Cephalometric norms of Nigerian children. Am J Orthod Dentofacial Orthop 2005;128(5):653-656

5 Davoody PR, Sassouni V. Dentofacial pattern differences between Iranians and American caucasians. Am J Orthod 1978;73(6):667-675

6 Sharma JN. Steiner's cephalometric norms for the Nepalese population. J Orthod 2011;38(1):21-31

7 Misch CE. Clinical indications for altering vertical dimension of occlusion. Objective vs subjective methods for determining vertical dimension of occlusion. Quintessence Int 2000;31(4):280-282

8 Asher F: Der totale Zahnersatz unter den Bedingungen des Gesichtsschdelaufbaus (Urban Schwarzenberg Verlag, Mnchen, 1971)

9 Ascher F. Reconstruction of the vertical dimension following loss of the natural dentition [Article in German]. Dtsch Zahnarztl Z 1973;28(1):18-29

10 Chaconas SJ, Gonidis D. A cephalometric technique for prosthodontic diagnosis and treatment planning. J Prosthet Dent 1986;56(5):567-574

11 Emad B, Abd ES, Osama AB, Magdy MB. A comparation between conventionally and cephalometrically determined vertical dimension of occlusion for completely edentulous patients. Ain Shams Dent J 2002;1:81-85

12 Strajnić L, Stanisić-Sinobad D, Marković D, Stojanović L. Cephalometric indicators of the vertical dimension of occlusion. Coll Antropol 2008;32(2):535-541

13 Wylie W. A syllabus in roentgenographic cephalometry. Am J Orthod 1957;43(6):465-466

14 Arunkumar KV, Reddy VV, Tauro DP. Establishment of cephalometric norms for the south Indian (karnataka) population based on Burstone's analysis. J Maxillofac Oral Surg 2010;9(2):127-133

15 Chopra R, Jain S, Jalili VP. Comparison of cephalometric norms of a cosmopolitan group in Indore to other population groups. J Indian Orthod Soc 2001;34:8-19

16 Al-Khateeb EA, Al-Khateeb SN. Anteroposterior and vertical components of class II division 1 and division 2 malocclusion. Angle Orthod 2009;79(5):859-866

17 Ricketts RM. Perspective in the clinical application of cephalometrics: the first fifty years. Angle Orthod 1981;51(2):115-150

18 Orthlieb JD. The curve of Spee: understanding the sagittal organization of mandibular teeth. Cranio 1997;15(4):333-340

19 Lahlou K, Bahoum A, Makhoukhi MB, Aalloula H. Comparison of dentoalveolar protrusion values in Moroccans and other populations. Eur J Orthod 2010;32(4):430-434

20 Kharbanda OP, Sidhu SS, Sundram KR. Cephalometric profile of north Indians: Tweed's analysis. Int J Orthod 1991;29(3-4):3-5 